\title{
PANICULITE MESENTÉRICA: ASPECTOS NA TOMOGRAFIA COMPUTADORIZADA*
}

\author{
Luiza Beatriz Melo Moreira', Ricardo Andrade Pinheiro², Alessandro Severo Alves de Melo ${ }^{3}$, \\ José Ricardo Duarte Alves ${ }^{4}$, Fabio Noro ${ }^{5}$, Edson Marchiori ${ }^{6}$
}

Resumo A paniculite mesentérica é um processo inflamatório que constitui o segundo estádio de uma doença rara e progressiva que envolve o tecido adiposo do mesentério. Entre os métodos radiológicos utilizados no diagnóstico estão os estudos baritados, a ultra-sonografia, a tomografia computadorizada e a ressonância magnética do abdome. A tomografia computadorizada tem importância tanto no diagnóstico e avaliação da extensão da doença quanto no planejamento do tratamento, e os aspectos tomográficos variam de acordo com o estádio da doença e se o componente predominante é inflamatório ou fibroso, tendo boa correlação com os achados da anatomopatologia. Os autores estudaram dez casos de paniculite mesentérica submetidos a tomografia computadorizada, sendo que em um deles foi realizada ressonância magnética. Em todos os casos o aspecto tomográfico era o de formação expansiva heterogênea, com densidade predominantemente adiposa, apresentando vasos ectasiados e bandas lineares com densidade de partes moles de permeio, localizada no mesentério.

Unitermos: Paniculite mesentérica. Radiologia. Tomografia computadorizada.

\begin{abstract}
Mesenteric panniculitis: computed tomography aspects.
Mesenteric panniculitis is an inflammatory process that represents the second stage of a rare progressive disease involving the adipose tissue of the mesentery. Imaging methods used in the diagnosis of mesenteric panniculitis include barium studies, ultrasonography, computed tomography and magnetic resonance imaging. Computed tomography is important for both, diagnosis and evaluation of the extension of the disease and treatment monitoring. Computed tomography findings may vary according to the stage of the disease and the amount of inflammatory material or fibrosis. There is also good correlation between the computed tomography and anatomical pathology findings. The authors studied 10 patients with mesenteric panniculitis submitted to computed tomography. Magnetic resonance imaging was also performed in one patient. In all patients, computed tomography revealed a heterogeneous mass in the mesentery with density of fat, interspersed with areas of soft tissue density and dilated vessels.

Key words: Mesenteric panniculitis. Radiology. Computed tomography.
\end{abstract}

\section{INTRODUÇÃO}

O espessamento fibroadiposo do mesentério intestinal é uma condição rara, de etiologia desconhecida. Vários termos

* Trabalho realizado no Departamento de Radiologia da Faculdade de Medicina da Universidade Federal Fluminense (UFF), Niterói, RJ, no Serviço de Radiodiagnóstico do Hospital Universitário Clementino Fraga Filho (HUCFF) da Universidade Federal do Rio de Janeiro (UFRJ), no Hospital de Clínicas de Niterói (HCN) e no Hospital Barra D'Or, Rio de Janeiro, RJ.

1. Médica Residente do Departamento de Radiologia da UFF.

2. Chefe do Serviço de Radiologia do Hospital Barra D'Or. 3. Mestre em Radiologia pela UFRJ, Médico do Serviço de Radiologia do HCN e do Hospital Barra D'Or.

4. Professor Adjunto do Departamento de Radiologia da UFF, Médico do Serviço de Radiologia do HCN e do Hospital Barra D'Or.

5. Médico do Serviço de Radiologia do Hospital Barra D'Or. 6. Professor Titular de Radiologia da UFF, Coordenado Adjunto do Curso de Pós-graduação em Radiologia da UFRJ.

Endereço para correspondência: Prof. Dr. Edson Marchiori. Rua Thomaz Cameron, 438, Valparaíso. Petrópolis, RJ, 25685-120. E-mail: edmarchiori@zipmail. com.br

Aceito para publicação em 20/2/2001 já foram utilizados para descrever o que parece ser o mesmo processo, incluindo mesenterite retrátil, paniculite mesentérica, manifestações mesentéricas da doença de Weber-Christian, lipogranuloma mesentérico, paniculite nodular sistêmica, esclerose subperitoneal multifocal, lipodistrofia mesentérica isolada, lipomatose mesentérica, entre outros ${ }^{(1-5)}$.

Embora a extensa terminologia possa determinar alguma confusão, esta condição pode ser considerada uma única doença com duas variantes patologicamente distintas. Nos casos em que a necrose de gordura e a inflamação predominam sobre a fibrose, o processo é definido como agudo ou subagudo e conhecido como lipodistrofia mesentérica e paniculite mesentérica, respectivamente; já quando a fibrose e a retração predominam, é considerado crônico e o resultado é a mesenterite retrátil ${ }^{(1-3,6)}$. A pre- sença de variados graus de fibrose torna o termo mesenterite esclerosante mais adequado $^{(\mathbf{1})}$. A paniculite mesentérica geralmente se caracteriza por episódios recorrentes de dor abdominal ${ }^{(\mathbf{1 , 6 - 8})}$.

Neste trabalho são discutidas as principais alterações encontradas na radiologia convencional, ultra-sonografia, tomografia computadorizada e ressonância magnética na paniculite mesentérica.

\section{CASUÍSTICA E MÉTOdos}

Foram reunidos, retrospectivamente, dez pacientes com paniculite mesentérica diagnosticada no período de maio de 1999 a dezembro de 2000, provenientes de três instituições hospitalares do Rio de Janeiro - Hospital Universitário Antônio Pedro (HUAP), Hospital de Clínicas de Niterói (HCN) e Hospital Barra D'Or -, com idades entre 42 e 76 anos, média de 
63,4 anos. Entre os pacientes estudados, cinco eram do sexo masculino e cinco, do sexo feminino.

Todos os pacientes foram submetidos a tomografia computadorizada de abdome em aparelhos Elscint (no HCN), Siemens Somaton Plus 4 (no Hospital Barra D'Or) e Siemens AR Star (no HUAP), com cortes de $5 \mathrm{~mm}$ e intervalos de 5 a 7,5 mm, do diafragma até a sínfise púbica, após a administração de contraste via oral e antes e após a administração de contraste iodado via venosa. Apenas um paciente realizou ressonância magnética. Comprovação histológica de necrose de gordura associada a processo inflamatório foi obtida em apenas um caso. Nos nove casos restantes, o diagnóstico de paniculite mesentérica foi feito com base nos aspectos tomográficos, em concordância com a literatura pesquisada, na qual se observa estreita correlação entre as imagens da tomografia computadorizada e o diagnóstico histológico ${ }^{(2,3)}$.

Nenhum dos pacientes tinha evidência de pancreatite, doença inflamatória intestinal ou de necrose de gordura extraabdominal (doença de Weber-Christian).

$\mathrm{Na}$ análise das tomografias computadorizadas os seguintes aspectos foram considerados: a) heterogeneidade das massas; b) predomínio da densidade de gordura; c) deslocamento das alças intestinais e/ou dos vasos; d) sinal do anel de gordura; e) pseudocápsula tumoral; f) linfonodomegalias; g) calcificações; h) áreas císticas; i) envolvimento retroperitoneal; j) complicações (compressão dos vasos mesentéricos, obstrução ureteral e obstrução intestinal).

\section{RESULTADOS}

Os aspectos tomográficos encontrados estão relacionados na Tabela 1 .
Todos os casos apresentavam formação expansiva heterogênea, com densidade predominantemente gordurosa e vasos ectasiados no seu interior (Figuras 1 a 4). Em oito casos ( $80 \%)$ havia deslocamento dos vasos (Figuras 1C e 4) e distorção das alças intestinais adjacentes (Figuras 3 e 4). Uma banda periférica com densidade de partes moles separando o mesentério normal do processo inflamatório (pseudocápsula tumoral) foi observada em sete pacientes (70\%) (Figura 1). A preservação da gordura adjacente aos vasos mesentéricos (sinal do anel de gordura) foi vista em apenas quatro casos (Figura 3).

Em nenhum dos dez casos analisados foram observados linfonodomegalia, calcificações, áreas císticas ou envolvimento retroperitoneal. Complicações como obstrução intestinal ou ureteral e compressão dos vasos mesentéricos também não foram evidenciadas.

\section{DISCUSSÃO}

A paniculite mesentérica geralmente envolve o mesentério do intestino delgado, especialmente na sua raiz, e ocasionalmente acomete o mesocólon; raramente há comprometimento da área peripancreática, omento e pelve ${ }^{(1,3,7)}$.

Sua etiologia permanece desconhecida, já tendo sido sugeridos como fatores causais infecção, trauma, cirurgia, isquemia mesentérica e alterações auto-imunes ${ }^{(\mathbf{1 , 3 - 5 , 8 , 9 )}}$. Esta condição é duas vezes mais comum nos homens do que nas mulheres, segundo relatos da maioria dos

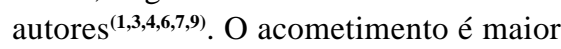
entre os indivíduos da quinta e sexta décadas de $v^{2} a^{(2-4,6,8)}$. Patel et al. ${ }^{(\mathbf{1})}$ atribuem a sua baixa freqüência em crianças à pouca quantidade de gordura mesentérica nestas.
Os critérios diagnósticos incluem: a) lesões com densidade de gordura configurando massas únicas, múltiplas ou difusas; b) confirmação histológica de necrose de gordura com infiltrado inflamatório e/ou infiltração com macrófagos; c) nenhuma evidência de pancreatite, doença inflamatória intestinal ou necrose de gordura extra-abdominal (doença de Weber-Christian). A fibrose pode estar presente, porém não é o achado dominante $^{(\mathbf{1 , 6 , 8})}$.

Os sinais e sintomas podem estar relacionados à inflamação ou ao efeito de massa em órgãos adjacentes, especialmente aprisionamento de alças intestinais ${ }^{(3)}$. Quando presentes, podem incluir dor abdominal (sintoma mais freqüente), geralmente associada a náuseas, vômitos, astenia, perda ponderal, febre baixa, diarréia ou constipação e sangramento

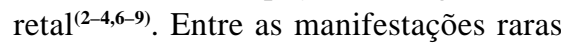
estão a febre de origem obscura, hemorragia gastrointestinal e obstrução dos vasos viscerais (veia cava inferior, veia mesentérica e ductos linfáticos $)^{(8)}$. Estes sintomas podem ser progressivos, intermitentes ou estar ausentes ${ }^{(2)}$, com duração média de seis meses ${ }^{(6)}$. Massa abdominal é freqüentemente palpável ${ }^{(2,4,6-9)}$.

Os achados laboratoriais são inespecíficos, podendo incluir leucocitose e velocidade de hemossedimentação discretamente elevada ${ }^{(6,8)}$. O diagnóstico geralmente é estabelecido por meio de laparotomia e biópsia ${ }^{(8)}$. O diagnóstico préoperatório acurado é infreqüente, em função da sintomatologia inespecífica ${ }^{(\mathbf{6})}$.

Os achados da patologia macroscópica variam do espessamento mesentérico às massas únicas ou múltiplas, de consistência endurecida ou fibroelástica, encapsuladas $^{(6)}$. Pode haver necrose de gordura, assim como variados graus de inflamação, hemorragia e fibrose ${ }^{(\mathbf{1 , 6})}$.

Tabela 1 Aspectos tomográficos.

\begin{tabular}{|c|c|c|c|c|c|c|c|c|c|c|c|c|}
\hline \multirow{2}{*}{$\begin{array}{c}\text { Aspectos na tomografia } \\
\text { computadorizada }\end{array}$} & \multicolumn{10}{|c|}{ Paciente $n^{0}$} & \multirow{2}{*}{ Total } & \multirow{2}{*}{ Porcentual } \\
\hline & 1 & 2 & 3 & 4 & 5 & 6 & 7 & 8 & 9 & 10 & & \\
\hline Massa heterogênea & + & + & + & + & + & + & + & + & + & + & 10 & $100 \%$ \\
\hline Deslocamento dos vasos & + & + & - & - & + & + & + & + & + & + & 8 & $80 \%$ \\
\hline Distorção das alças intestinais & + & + & - & - & + & + & + & + & + & + & 8 & $80 \%$ \\
\hline Pseudocápsula tumoral & + & - & + & - & + & + & + & - & + & + & 7 & $70 \%$ \\
\hline Sinal do anel de gordura & - & + & + & - & - & - & - & - & + & + & 4 & $40 \%$ \\
\hline
\end{tabular}




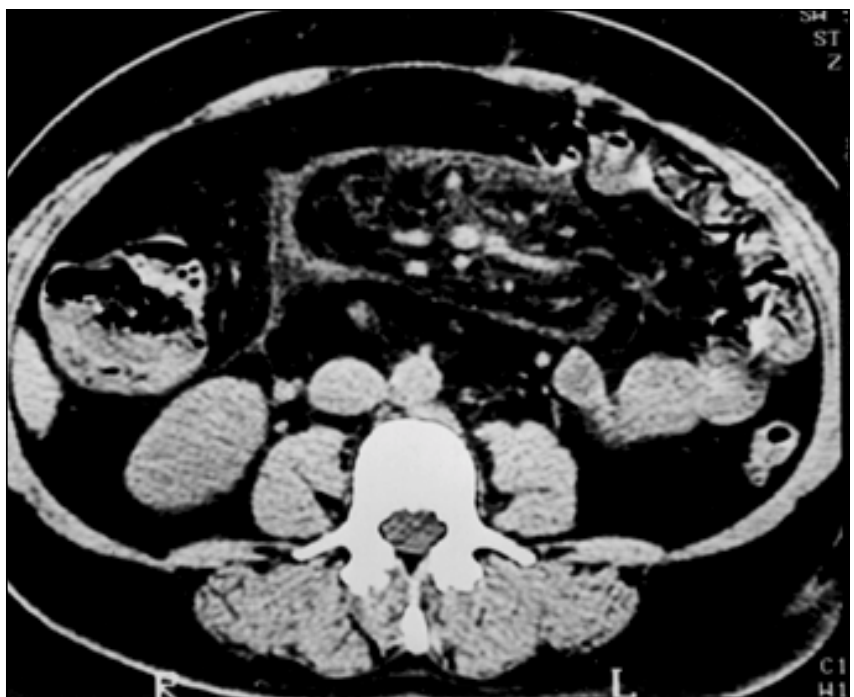

A

Figura 1. Em A e $\mathbf{B}$, imagens de tomografia computadorizada do abdome sem contraste venoso demonstram formação expansiva heterogênea, com densidade predominantemente gordurosa, apresentando vasos ectasiados no seu interior, com limites parcialmente bem definidos por cápsula espessa, localizada no mesentério, em topografia mesogástrica. Bandas lineares com densidade de partes moles podem ser observadas de permeio à lesão, representando reação fibrosa no interior do processo inflamatório do tecido adiposo. Em C, após a administração do meio de contraste iodado, há impregnação heterogênea da lesão, mais evidente em sua cápsula.

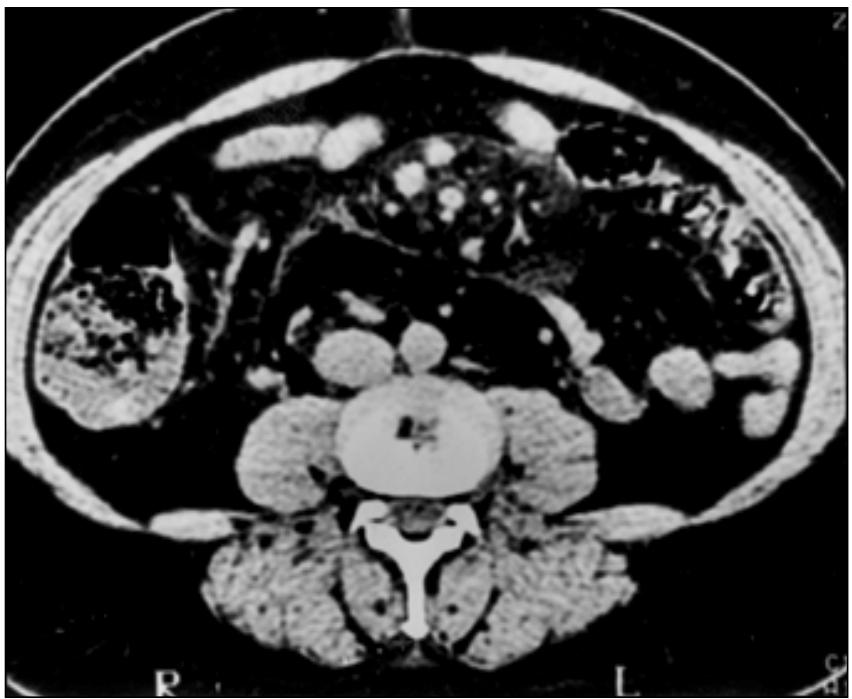

B

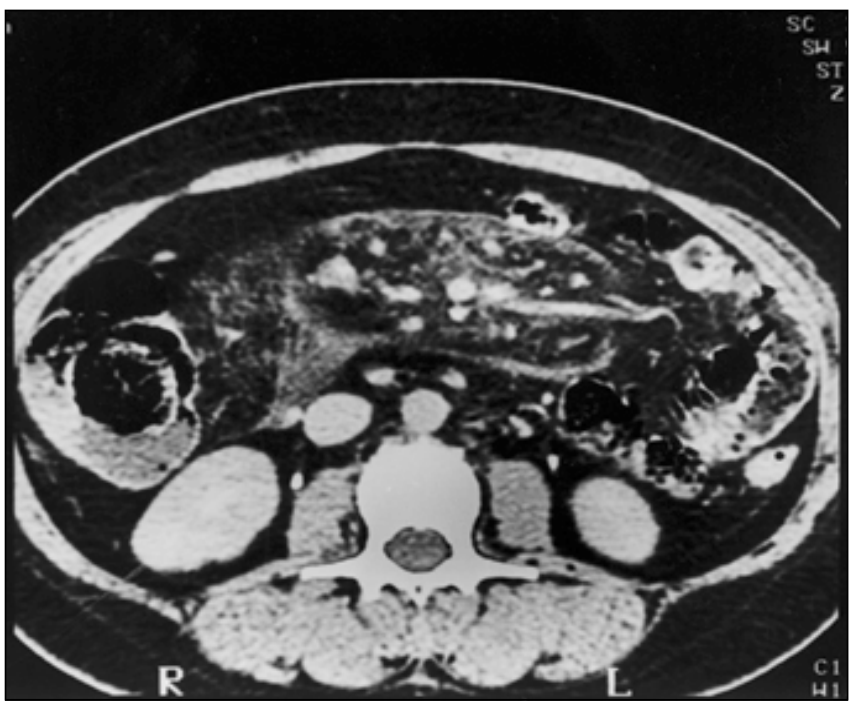

C

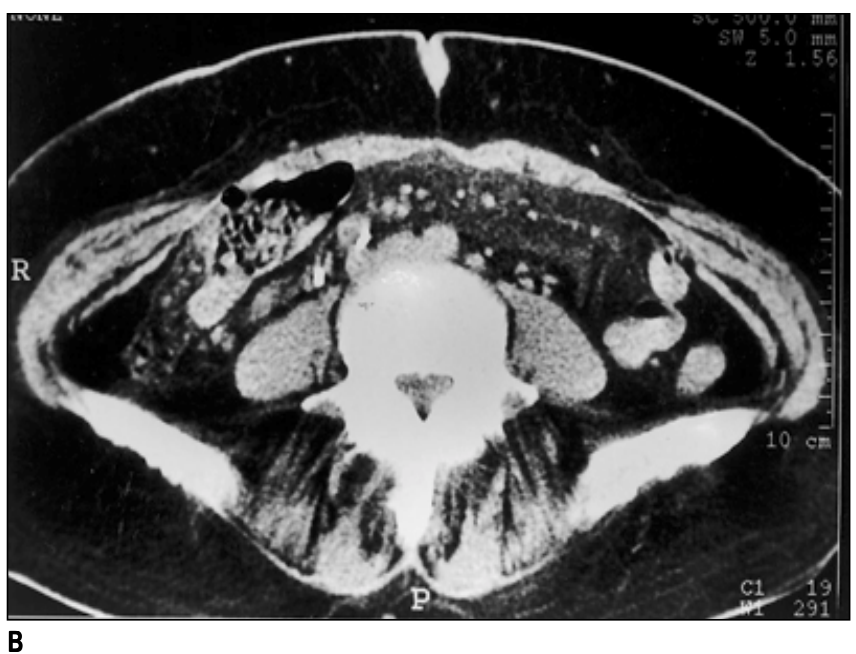

A

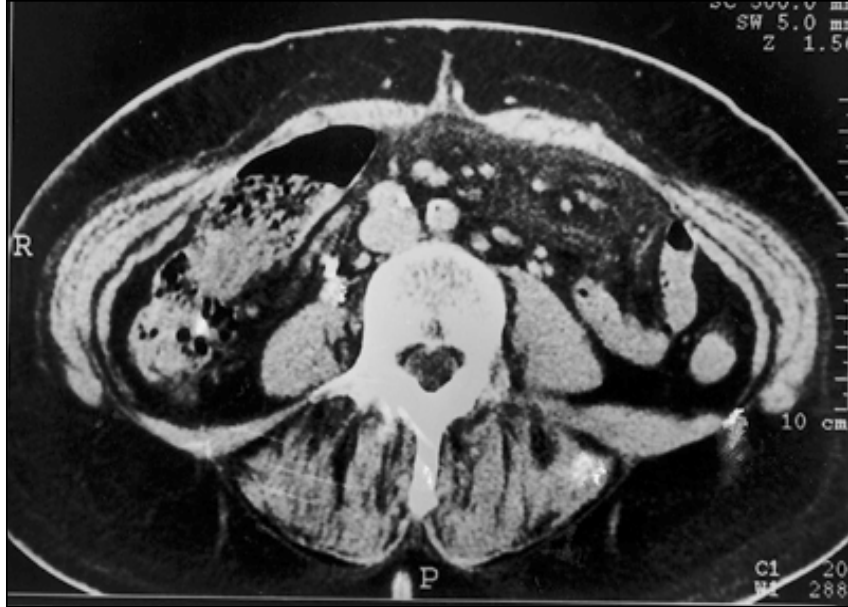

Figura 2. Cortes tomográficos obtidos após a injeção endovenosa de contraste iodado mostram formação expansiva de limites imprecisos, hipodensa e heterogênea, caracterizada pela presença de grande componente adiposo, cuja densidade está discretamente elevada (gordura espessada), e vasos dilatados no seu interior. A lesão está localizada no mesentério, na região do mesogástrio. 


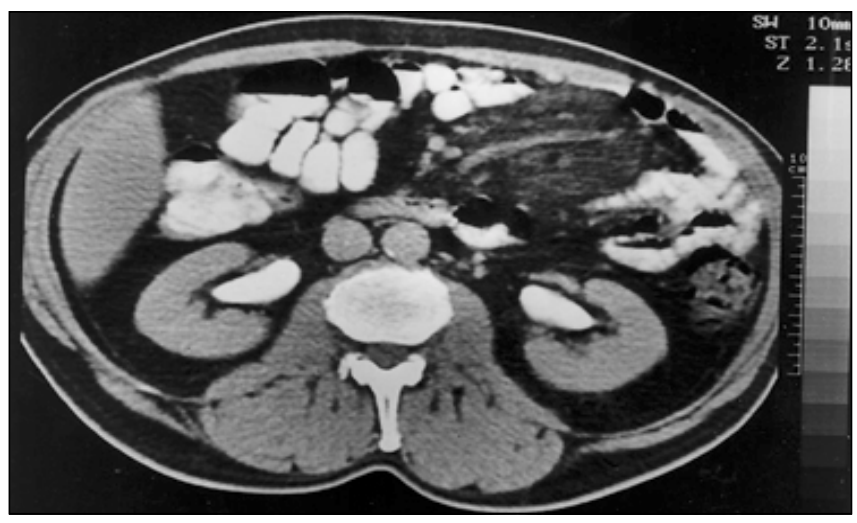

Figura 3. Tomografia computadorizada do abdome após a administração do meio de contraste evidencia área mesentérica de tecido gorduroso espessado, com vasos intumescidos no seu interior, determinando compressão sobre as alças intestinais adjacentes.
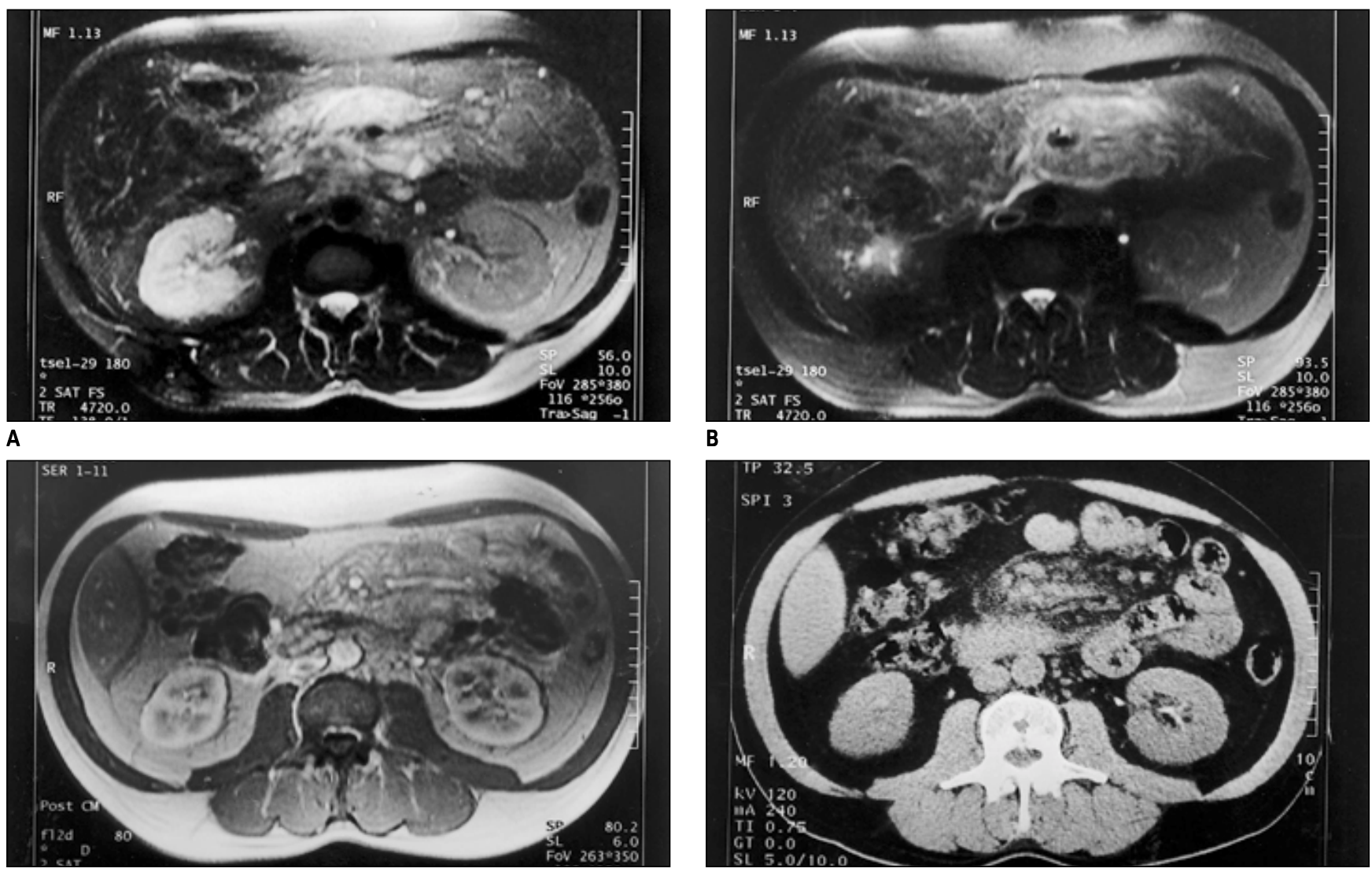

C

\section{B}

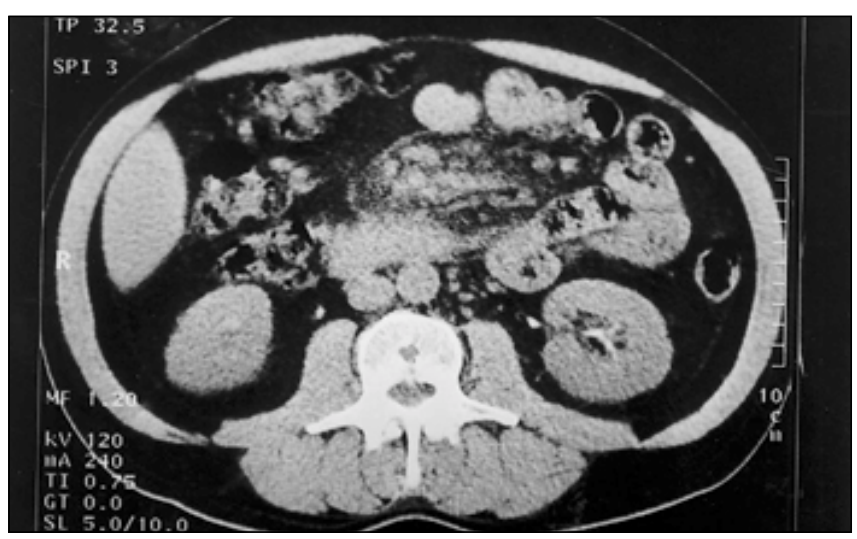

D

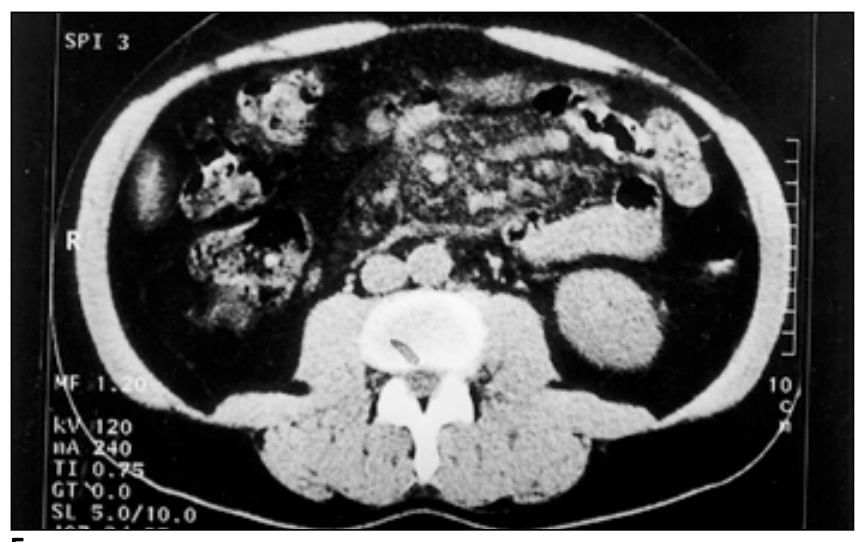

Figura 4. Em A e $\mathbf{B}$, imagens de ressonância magnética do abdome no plano axial com a técnica TSE T2 com supressão de gordura demonstram formação expansiva mesentérica bem delimitada, de sinal elevado, com afastamento das alças intestinais adjacentes e vasos mesentéricos ectasiados no seu interior. Em C, imagem obtida com a técnica gradiente-eco em T1 com contraste evidencia opacificação das estruturas vasculares e o rechaço das alças intestinais. Em $\mathbf{D}$ e $\mathbf{E}$, cortes tomográficos do mesmo paciente mostram o aspecto tomográfico da paniculite. 
No exame histopatológico, calcificações estão freqüentemente presentes, provavelmente como resultado da necrose de gordura $^{(4)}$, sendo raramente vistas na

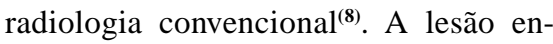
volve a gordura do mesentério e da submucosa da alça intestinal de delgado, geralmente se estendendo às camadas muscular e submucosa do intestino, preservando a mucosa. O processo pode provocar obstrução dos linfáticos mesentéricos, resultando em edema de submucosa e linfangiectasia ${ }^{(\mathbf{1 , 4})}$. Os vasos mesentéricos freqüentemente exibem estreitamento luminal, levando ao comprometimento da função gastrointestinal normal e ascite quilosa ${ }^{(\mathbf{1 , 4})}$.

A mesenterite retrátil já foi descrita associada a fibrose retroperitoneal, linfoma, síndrome de Gardner, lesão ulcerativa do reto, nódulos ovarianos semelhantes a sarcomas, mesotelioma torácico, vasculites, doença granulomatosa, doença reumática, doenças malignas e pancreatite $^{(2,3)}$.

As principais complicações estão relacionadas a fibrose progressiva, encurtamento do mesentério e compressão de vasos mesentéricos, com obstrução parcial ou completa, levando à isquemia, e podem requerer cirurgia ${ }^{(3)}$. A obstrução ureteral está associada ao envolvimento retroperitoneal $^{(3)}$. Quando há obstrução intestinal, esta é mais freqüente no intestino delgado ${ }^{(\mathbf{1})}$.

Entre os achados radiológicos mais freqüentes nos estudos baritados estão a separação, fixação e angulação das alças intestinais, sugerindo comprometimento da serosa ${ }^{(\mathbf{3 , 4})}$. As pregas intestinais podem estar espessadas por causa da extensão do processo inflamatório à submucosa ou edema por obstrução linfática ${ }^{(3,4)}$. A preservação do padrão mucoso é um importante sinal na diferenciação entre este processo e uma neoplasia primária ${ }^{(3)}$. O estreitamento luminal parcial é outro achado comum na mesenterite retrátil, porém a estenose completa é incomum ${ }^{(4)}$. Sabaté et al. ${ }^{(3)}$ descreveram acometimento das alças intestinais mais evidente nos pacientes com mesenterite retrátil do que naqueles com paniculite mesentérica ${ }^{(3)}$. Ocasionalmente, o cólon pode estar envolvido, com estreitamento e rigidez ${ }^{(4)}$.
O aspecto sonográfico da paniculite mesentérica é variável e inespecífico, tendo sido descritas tanto massas heterogêneas predominantemente hiperecóicas quanto massas hipoecóicas ${ }^{(6,7)}$. A ultrasonografia "color"-Doppler pode mostrar claramente os vasos mesentéricos no interior das massas ${ }^{(7)}$.

A tomografia computadorizada é utilizada no diagnóstico e na avaliação da eficácia do tratamento ${ }^{(\mathbf{3 , 8})}$. Os achados tomográficos dependem do estádio da doença e do componente predominante (inflamatório, fibroso ou adiposo) ${ }^{(5)} \mathrm{e}$ apresentam boa correlação com os achados patológicos ${ }^{(3,7)}$. A paniculite mesentérica geralmente se apresenta como massas heterogêneas com grande componente de gordura, cuja densidade se encontra discretamente elevada, associada a bandas lineares com densidade de partes moles de permeio $^{(\mathbf{3 , 4})}$, sem invasão $\operatorname{vascular}^{(7-9)}$. A elevação da densidade da gordura ocorre em consequiência ao processo inflamatório do tecido adiposo, e as bandas lineares correspondem à retração fibrosa ${ }^{(3)}$. Ao contrário, as massas da mesenterite retrátil têm aspecto homogêneo e predomínio de densidade de partes moles, refletindo a natureza fibrótica da doença. Anormalidades vasculares foram descritas tanto na radiologia como na anatomopatologia ${ }^{(3,8)}$. Calcificações associadas a necrose de gordura são achados raros nesta doença, porém, quando presentes, são facilmente detectáveis na tomografia computadorizada ${ }^{(3,4,9)}$.

Sabaté et $a l .{ }^{(3)}$ realizaram um estudo retrospectivo de 17 casos de mesenterite esclerosante patologicamente comprovados, incluindo o período de 1981 a 1997 , sendo 12 com paniculite mesentérica e cinco com mesenterite retrátil. Em três pacientes foi observada discreta linfonodomegalia retroperitoneal e mesentérica $(17,6 \%)$, cujo exame histológico revelou alterações inflamatórias e descartou a coexistência de doença linfoproliferativa. Nesse estudo, os autores observaram dois aspectos que sugerem o diagnóstico tomográfico de paniculite mesentérica: o sinal do anel de gordura e a presença de pseudocápsula tumoral. No primeiro foi observada a preservação da gordura próxima aos vasos mesentéricos (em nove dos 12 pacientes). Os autores enfatizam a sua importância, não apenas para o diagnóstico mas também na exclusão de outros tumores lipomatosos, especialmente o lipossarcoma lipogênico. A pseudocápsula consiste de uma banda periférica com densidade de partes moles que separa o mesentério normal do processo inflamatório (relatada em seis dos 12 casos estudados). Estes aspectos desaparecem quando a paniculite mesentérica "evolui" para a mesenterite retrátil, o que ainda não foi explicado $^{(3)}$

A paniculite mesentérica pode manifestar-se sob a forma de massa cística multiloculada, sendo que tais áreas císticas se devem provavelmente a canais linfáticos dilatados, em decorrência da obstrução linfática secundária à própria paniculite $^{(6)}$.

A ressonância magnética tem papel importante no diagnóstico da mesenterite esclerosante (paniculite mesentérica ou mesenterite retrátil), já que permite melhor caracterização do tecido e acesso não-invasivo a vasos de médio a grande calibre $^{(4)}$. As massas podem exibir uma variedade de sinais em função de seu componente gorduroso ou fibroso ${ }^{(4,5)}$. Nas seqüências ponderadas em T1 a lesão tende a apresentar sinal intermediário, já naquelas ponderadas em T2 o padrão de sinal pode variar de acordo com o tecido predominante, ou seja, se houver fibrose intensa haverá hipossinal, se houver predomínio de processo inflamatório e gordura residual o sinal será mais elevado $^{(5)}$. Sinal baixo sugere reação fibrótica madura ${ }^{(4)}$. A ressonância magnética pode mostrar a separação de alças intestinais e a presença de vasos mesentéricos no interior da massa, que demonstra "flow voids" (falha de fluxo) ${ }^{(5)}$. Imagens ponderadas em T2 ou com supressão de gordura podem auxiliar na distinção entre proliferação fibroadiposa e tumores malignos, como os linfomas e as metástases $^{(\mathbf{4})}$.

O diagnóstico diferencial da mesenterite retrátil inclui carcinomatose, mesotelioma peritoneal, linfoma, tumor carcinóide e tumor desmóide ${ }^{(\mathbf{1 , 3 , 8 , 9 )}}$. No caso da lipodistrofia mesentérica, podem ser incluídos no diagnóstico diferencial a fibromatose mesentérica, pseudotumores 
inflamatórios, fibrose retroperitoneal idiopática, tumor mesentérico maligno, metástases e doenças granulomatosas ${ }^{(\mathbf{8})}$. Em relação à paniculite mesentérica, o diagnóstico diferencial pode ser feito com pancreatite necrotizante, leiomiossarcoma, doença de Crohn com proliferação fibroadiposa, abscessos, hematomas e neoplasias adiposas (lipossarcoma, mielolipoma, angiomiolipoma, teratoma), além dos já citados para a mesenterite retrátil ${ }^{(3,7)}$. O lipossarcoma lipogênico possui diagnóstico diferencial difícil, clínica e radiologicamente, sendo necessário, na maioria dos casos, biópsia cirúrgica ou laparoscópica ${ }^{(3)}$.

Presença de linfonodomegalia retroperitoneal foi sugerida como achado contrário à mesenterite retrátil, embora tenha sido encontrada em três dos 17 pacientes estudados por Sabaté et al. ${ }^{(3)}$. Pacientes com paniculite mesentérica podem evoluir com linfoma associado durante o seguimento $^{(\mathbf{1})}$.

Há três formas de evolução: a) resolução parcial ou completa do processo inflamatório; b) estabilização da condição; c) curso agressivo, com desfecho fatal em algumas vezes ${ }^{(3)}$.

O prognóstico geralmente é bom, sem tratamento específico, embora ocasionalmente haja recorrências ${ }^{(\mathbf{1 , 3 , 4 , 6})}$. Alguns autores sugerem que as formas colônicas tenham evolução mais agressiva, sendo necessária a cirurgia mais freqüentemente do que nas formas que envolvem o intestino delgado ${ }^{(3)}$.

Nenhum tratamento é considerado totalmente eficaz. Enquanto nas formas indolentes da doença não é necessário tratamento específico ${ }^{(\mathbf{1}, \mathbf{3}, \mathbf{4}, \mathbf{8}}$, nos casos mais avançados ou progressivos têm sido usados corticosteróides e imunossupressores $^{(\mathbf{1 , 3 , 4 , 8 )}}$. A abordagem cirúrgica deve ser limitada a exploração com biópsia e procedimentos de interposição de alças nos casos de obstrução intestinal ${ }^{(3,4,8)}$, reservando-se a ressecção para os casos considerados altamente suspeitos para a malignidade. A cirurgia radical não é aconselhada, já que alguns pacientes submetidos à ressecção tiveram recorrências, e outros sem ressecção tiveram remissões completas documentadas ${ }^{(6)}$.

\section{REFERÊNCIAS}

1. Patel N, Saleeb SF, Teplick SK. General case of the day. Mesenteric panniculitis with extensive inflammatory involvement of the peritoneum and intraperitoneal structures. RadioGraphics 1999;19: 1083-5.

2. Daskalogiannaki M, Voloudaki A, Prassopoulos P, et al. CT evaluation of mesenteric panniculitis: prevalence and associated diseases. AJR 2000;174: 427-31.

3. Sabaté JM, Torrubia S, Maideu J, Franquet T, Monill JM, Pérez C. Sclerosing mesenteritis: imaging findings in 17 patients. AJR 1999;172:625-9.

4. Kronthal AJ, Kang YS, Fishman EK, Jones B, Kuhlman JE, Tempany CMC. MR imaging in sclerosing mesenteritis. AJR 1991;156:517-9.

5. Fujiyoshi F, Ichinari N, Kajiya Y, et al. Retractile mesenteritis: small-bowel radiography, $\mathrm{CT}$, and MR imaging. AJR 1997;169:791-3.

6. Johnson LA, Longacre TA, Wharton KA Jr, Jeffrey RB. Multiple mesenteric lymphatic cysts: an unusual feature of mesenteric panniculitis (sclerosing mesenteritis). J Comput Assist Tomogr 1997; 21:103-5.

7. Catalano O, Cusati B. Sonographic detection of mesenteric panniculitis: case report and literature review. J Clin Ultrasound 1997;25:141-4.

8. Hemaidan A, Vanegas F, Alvarez OA, Arroyo MA, Lee M. Mesenteric lipodystrophy with fever of unknown origin and mesenteric calcifications. South Med J 1999;92:513-6.

9. Ueda D, Chiba S. Retractile mesenteritis in a 12year-old girl: CT findings. Pediatr Radiol 1997; 27:342-4. 